\title{
Induction of apoptosis by D-limonene is mediated by inactivation of Akt in LS174T human colon cancer cells
}

\author{
SHU-SHENG JIA ${ }^{1}$, GUANG-PENG XI ${ }^{2}$, MING ZHANG ${ }^{1}$, \\ YAN-BO CHEN $^{1}$, BO LEI ${ }^{1}$, XIN-SHU DONG ${ }^{3}$ and YAN-MEI YANG ${ }^{2}$ \\ ${ }^{1}$ Department of Breast Surgery, The Third Affiliated Hospital of Harbin Medical University, Harbin; \\ ${ }^{2}$ Cancer Research Institute, Harbin Medical University, Harbin; ${ }^{3}$ The Fourth Affiliated \\ Hospital of Harbin Medical University, Harbin, Heilongjiang 150081, P.R. China
}

Received August 20,2012; Accepted October 3, 2012

DOI: $10.3892 / o r .2012 .2093$

\begin{abstract}
D-limonene is recognized as a potential chemotherapeutic agent, however, the details of this mechanism remain unclear. In this study, we investigated the effects of D-limonene on colon cancer cell viability and its potential mechanism of action in vitro. After $48 \mathrm{~h}$ of treatment, D-limonene suppressed the viability of LS174T cells in a dosedependent manner and caused a dose-dependent apoptotic cell death. D-limonene activated caspase-3 and -9 and PARP cleavage in a dose-dependent manner. Moreover, an increase in Bax protein and cytosol cytochrome $c$ from mitochondria and a decrease in Bcl-2 protein were observed following treatment with D-limonene. In addition, D-limonene decreased the levels of p-Akt (Ser473), p-Akt (Thr308) and p-GSK-3 $\beta$ (Ser9), suggesting that D-limonene induced apoptosis via the mitochondrial death pathway and the suppression of the PI3K/ Akt pathway.
\end{abstract}

\section{Introduction}

D-limonene (1-methyl-4-isopropyl-cyclohexene) is a cyclic monoterpene and a major constituent in essential oils of orange, lemon, mandarin, lime, grapefruit and several other plants. It is listed in the Code of Federal Regulations as generally recognized as safe (GRAS) for a flavoring agent and can be found in common food items such as fruit juices, soft drinks, baked goods, ice cream and pudding (1). One epidemiological

Correspondence to: Dr Yan-Mei Yang, Cancer Research Institute, Harbin Medical University, 6 Baojian Road, Nangang, Harbin, Heilongjiang 150081, P.R. China

E-mail: yangym0916@163.com

Professor Xin-Shu Dong, The Fourth Affiliated Hospital of Harbin Medical University, Harbin, Heilongjiang 150081, P.R. China

E-mail: dxs82132079@126.com

Key words: D-limonene, Akt, apoptosis study suggested that citrus peel consumption, the major source of dietary D-limonene, might have a potential preventive effect on squamous cell carcinoma (2). In chemically-induced rodent tumor models, D-limonene has been reported to have chemopreventive activity at both the initiation and promotion/progression phases (3-8). Evidence from a phase I clinical trial showed that D-limonene is well tolerated and may have clinical activity in cancer patients (9). While these observations are of interest, a better understanding of the underlying action mechanisms of D-limonene is necessary to confirm its effectiveness as a potential chemopreventive and treatment agent.

Several mechanisms may account for the anticancer effect of D-limonene. The chemopreventive activity of D-limonene during the initiation phase of carcinogenesis might be attributed to the induction of phase I and phase II carcinogen-detoxifying enzymes, which are known to be responsible for detoxification of carcinogen (10-12). A previous report indicated that the topical treatment of D-limonene inhibits the Ras-ERK signaling pathway, inflammation and oxidative stress as well as the induction of pro-apoptotic state in the TPA-mediated promotion of DMBA-induced skin cancer in a mouse model (13). In vivo and in vitro studies demonstrated that the chemotherapeutic activity of D-limonene might be attributed to the induction of apoptosis (14-16). Ji et al found that the limoneneinduced apoptotic death of human leukemia cells involves the increase in Bax protein expression, the release of cytochrome $c$ from mitochondria and the activation of caspase, suggesting that the mitochondria-mediated intrinsic death pathway may play a major role in limonene-induced death (16).

The serine/threonine protein kinase (Akt, a member of the PI3K pathway) is involved in widely divergent cellular processes including apoptosis and cell proliferation (17). The aberrant activation of phosphoinositide 3-kinase (PI3K)/AKT has been documented as a frequent occurrence in human cancer, including colorectal cancer (18-20). Furthermore, abnormal activation of the PI3K/AKT pathway rendered these cells less sensitive to apoptosis stimuli $(21,22)$ and inhibition of this pathway should provide a therapeutic approach for cancer (23). Once activated, however, Akt promotes the cell survival partially by phosphorylation and inactivation of several pro-apoptotic proteins, including GSK-3 (24), BAD (25) and 
caspase-9 (26). Although it has been reported that geraniol, an acyclic dietary monoterpene, potently induced apoptosis and autophagy by inhibition of AKT signaling (27), the effect of D-limonene on Akt signaling remains unclear.

One phase I study conducted by Vigushin et al indicated that three individuals with colorectal carcinoma, while on D-limonene at dose of $0.5-1 \mathrm{~g} / \mathrm{m}^{2}$ per day, were able to suspend progression of the disease for over six months (9). This clinical trial strongly suggested that D-limonene could be an efficient therapeutic agent for colorectal cancer. The mechanism by which D-limonene inhibits the viability of colorectal cancer cells has yet to be established, therefore, we addressed this in the present study.

\section{Materials and methods}

Materials. D-limonene was purchased from Sigma Chemical Co. (St. Louis, MO, USA). Antibodies against total Akt, phospho-Akt (Ser473), phospho-Akt (Thr308), phosphoGSK-3 $\beta$ (Ser9), poly(ADP-ribose)polymerase (PARP), cleaved PARP, pro-caspase-3, cleaved caspase-3, pro-caspase- 9 , cleaved caspase- 9 , pro-caspase- 8 , cleaved caspase- 8 and $\beta$-actin were purchased from Cell Signaling Technology (Beverly, MA, USA). Antibodies against Bcl-2 and Bax were purchased from Santa Cruz Biotechnology (Santa Cruz, CA, USA). Horseradish peroxidase (HRP)-conjugated secondary antibodies were purchased from Cell Signaling Technology. Annexin V-fluorescein isothiocyanate (FITC) labeled apoptosis detection kit was obtained from Baosai Biological Technology Co., Ltd. (Beijing, China).

Cell culture. The LS174T human colon cancer cell line (Heilongjiang Cancer Institute, China) was maintained in RPMI-1640 medium at $37^{\circ} \mathrm{C}$ in a $5 \% \mathrm{CO}_{2}$ atmosphere. All cell samples used were in the logarithmic growth phase.

Cell viability assay. Effect of D-limonene on the viability of LS174T cells was performed using 3-(4,5-dimethylthiazol2-yl)-2,5-diphenyltetrazolium bromide (MTT) conversion assay. Briefly, LS174T cells were plated into a 24-well plate and cultured in the presence of varying concentrations of D-limonene for $48 \mathrm{~h}$. Thereafter, $10 \mu \mathrm{l}$ of MTT $(5 \mathrm{mg} / \mathrm{ml})$ was added to each well and the cells were incubated at $37^{\circ} \mathrm{C}$ for another $4 \mathrm{~h}$. Following removal of the medium, the cells were lysed in $100 \mu$ l of dimethylsulfoxide (DMSO). Then, the optical density (OD) was measured at $490 \mathrm{~nm}$ by a microplate reader (Bio-Rad Laboratories, Hercules, CA, USA). The following formula was used: relative percentage of cell viability $=(\mathrm{OD}$ of the experimental sample/OD of the control group) $\mathrm{x} 100 \%$.

Measurement of apoptosis by Annexin V-FITC staining. Apoptosis was determined by staining cells with Annexin VFITC staining. Briefly, after various treatments, both floating and trypsinized adherent LS174T cells were collected. Then the cell pellets were incubated with $5 \mu \mathrm{l}$ propidium iodide (PI), $10 \mu 1$ Annexin V-FITC for 20 min in the dark at room temperature and the samples were analyzed by flow cytometry.

Western blot analysis. Cytosolic extracts were prepared for cytochrome $c$ according to the instructions for the Nuclear

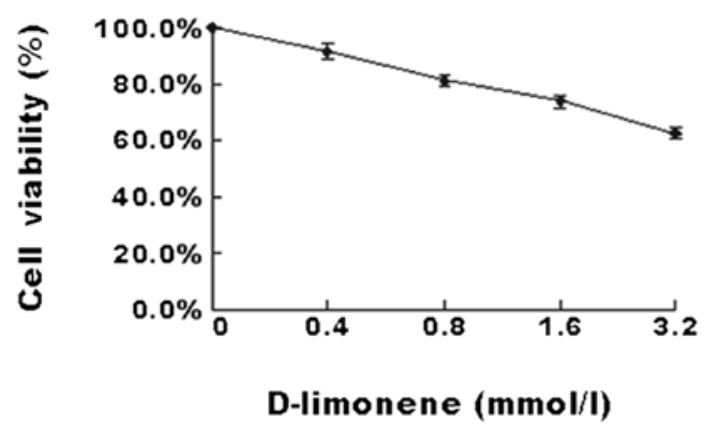

Figure 1. Effect of D-limonene treatment on cell viability. Cell viability analysis: LS174T cells were treated with D-limonene for $48 \mathrm{~h}$ and cell viability was determined by MTT assay. Data shown are the means \pm SD from 3 separate experiments in quintuplicate.

and Cytoplasmic Protein Extraction kit (active motif 40010). Total cell extracts were prepared by resuspending cells in cold lysis buffer (Beyotime, Jiangsu, China). The supernatants were then collected by centrifugation at 12,000 $\mathrm{x}$ g for $5 \mathrm{~min}$. Equal amounts of lysate were loaded on a $10 \%$ SDS-polyacrylamide gel. After electrophoresis, proteins were transferred to a PVDF membrane and the blots were probed by corresponding primary antibodies, followed by incubation with HRP-conjugated secondary antibodies. The positive bands representing protein were visualized by chemiluminescent reagents (EZ-ECL; Biological Industries, Kibbutz Beit Haemek, Israel).

Statistical analysis. Data are expressed as the means \pm SD of 3 repeated experiments. The one way analysis of variance (ANOVA) was used for statistical analyses. $\mathrm{P}<0.05$ was considered to indicate statistically significant differences. All experiments were performed at least 3 times independently.

\section{Results}

D-limonene decreases the cell viability of LS174T cells. To investigate the effect of D-limonene on the viability of colon cancer cells, LS174T cells were treated with various concentrations of D-limonene for $48 \mathrm{~h}$. The results of the MTT assay (Fig. 1) demonstrated that D-limonene significantly inhibited cell viability in a dose-dependent manner.

D-limonene induces apoptosis of LS174T cells. To determine whether D-limonene inhibits the viability of LS174T cells via induction of apoptosis, LS174T cells were treated with various concentrations of D-limonene for $48 \mathrm{~h}$ and apoptosis was quantified using flow cytometry with Annexin V/PI dual staining. The results revealed that D-limonene resulted in a dose-dependent induction of apoptosis in LS174T cells (Fig. 2). These results suggest that D-limonene inhibited the viability of LS174T cells by inducing apoptosis in a dose-dependent manner.

D-limonene induces caspase activation in LS174T cells. To investigate whether or not the observed apoptosis was due to caspase activation, the cell extracts were obtained after treatments and processed for western blot analysis. The 

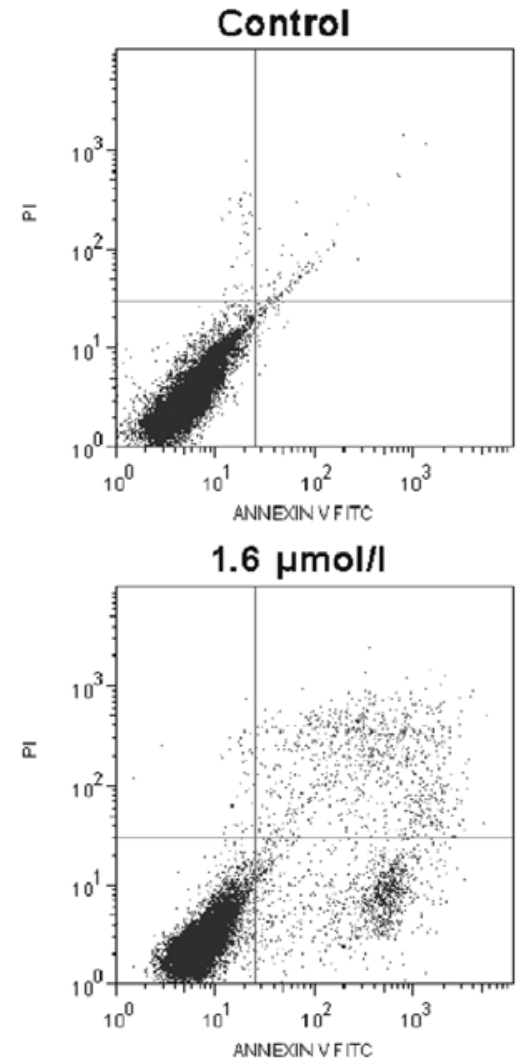

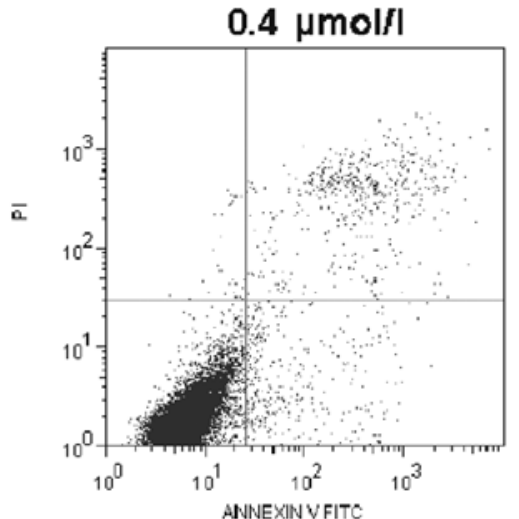

$3.2 \mu \mathrm{mol} / \mathrm{l}$

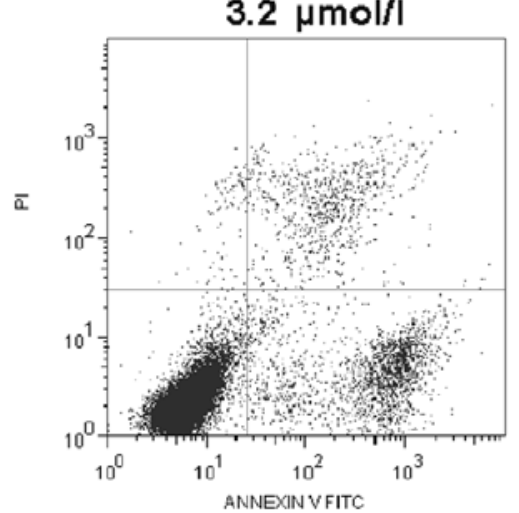

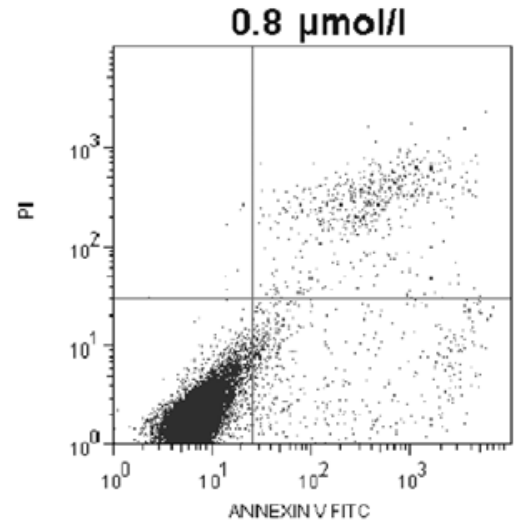

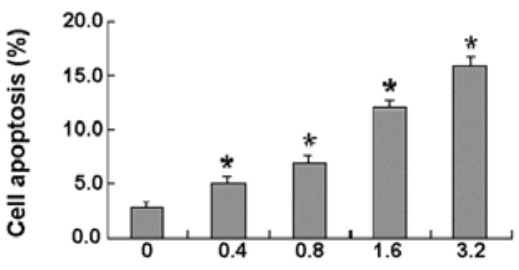

D-limonene ( $\mathrm{mmol} / \mathrm{l})$

Figure 2. Effect of D-limonene treatment on apoptosis. LS174T cells were treated with D-limonene for $48 \mathrm{~h}$ and apoptosis was determined by flow cytometry as detailed in Materials and methods. The cells were labeled with Annexin-FITC and PI. Annexin V-positive cells are considered apoptotic cells and their percentage is indicated. Data from 3 independent experiments are shown. " $\mathrm{P}<0.05$ vs. control.

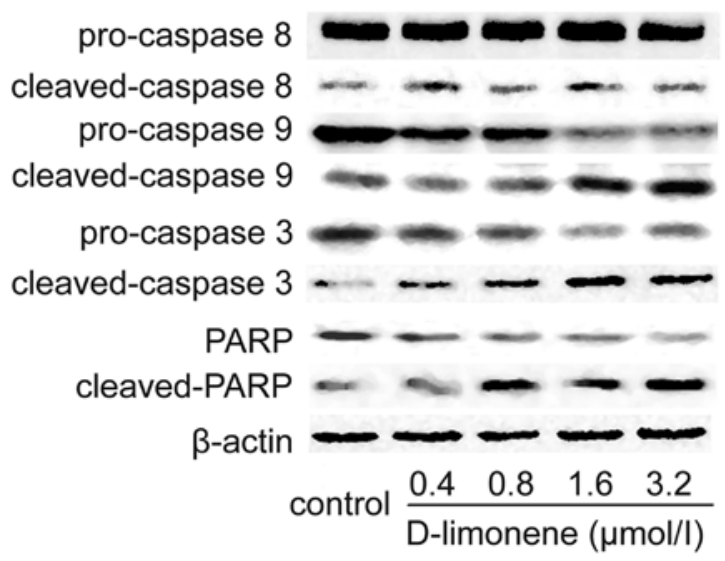

Figure 3. Effect of D-limonene on caspase activation in LS174T cells. LS174T cells were incubated with D-limonene for $48 \mathrm{~h}$ and the proteins were analyzed by western blotting with antibodies against pro-caspase-3, cleaved caspase-3, pro-caspase-9, cleaved caspase-9, PARP and cleaved PARP.

changes in protein levels in the LS174T cells treated with D-limonene were determined using western blot analysis. After $48 \mathrm{~h}$, D-limonene treatment resulted in partial cleavage of pro-caspase-3, and -9 , whereas it had no significant effect on caspase-8 expression (Fig. 3), which indicated that D-limonene could increase the cleavage maturation of caspase- 3 and caspase-9. Meanwhile, the cleavage of PARP, an executioner

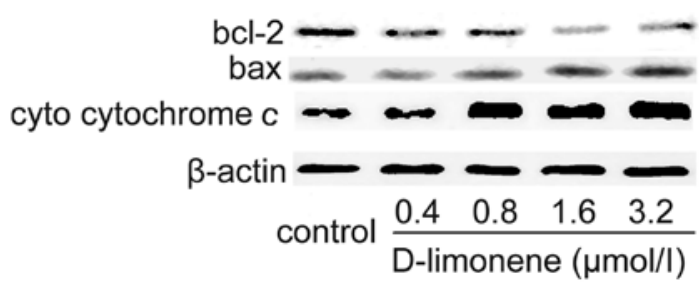

Figure 4. Effect of D-limonene on the expression of Bcl-2, Bax protein and cytosol cytochrome $c$ in LS174T cells. LS174T cells were incubated with D-limonene for $48 \mathrm{~h}$; then, the total proteins were analyzed by western blotting with antibodies against $\mathrm{Bcl}-2$ and Bax, the cytosol protein was also analyzed by western blotting with antibodies against cytochrome $c$.

caspase substrate, was also detected using western blot analysis. Similarly, D-limonene treatment resulted in cleavage of PARP from 116 to $89 \mathrm{kDa}$ (Fig. 3). Therefore, these results suggest that D-limonene-induced apoptosis is partly mediated through the mitochondrial pathway.

$D$-limonene regulates the expression of $B c l-2$ and Baxprotein. To confirm the involvement of the mitochondrial pathway of apoptosis, the expression of Bax and Bcl-2 in the LS174T cells treated with D-limonene were determined using western blot analysis. D-limonene treatment resulted in an upregulation of Bax and a slight downregulation of Bcl-2 (Fig. 4), which 


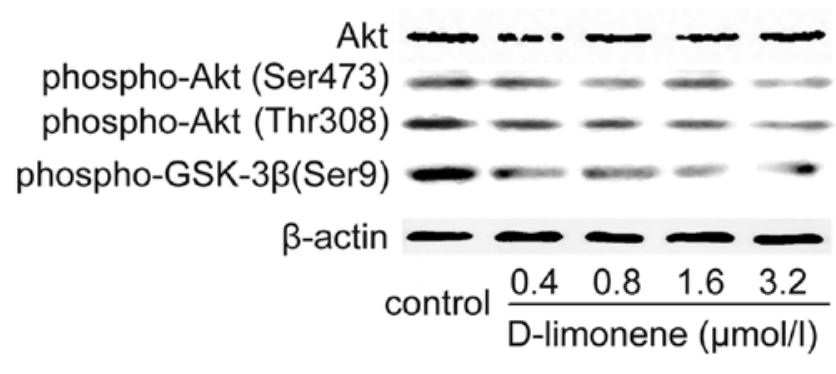

Figure 5. Effect of D-limonene on the activation and expression of Akt proteins. Exponentially growing cells were treated with D-limonene for $48 \mathrm{~h}$ and the Akt, phospho-Akts, and phospho-GSK3 $\beta$ (ser9) were evaluated using western blot analysis.

resulted in an increase in the ratio of Bax to $\mathrm{Bcl}-2$, therefore favored apoptosis. Since the release of cytochrome $c$ from the mitochondria triggers the activation of pro-caspase 9, we measured cytosol cytochrome $c$. D-limonene significantly increased the level of cytosol cytochrome $c$ (Fig. 4), which further suggested that D-limonene-induced apoptosis is partly mediated through the mitochondrial pathway.

D-limonene inhibits phosphorylation of Akt in LS174T cells. Akt is reported to promote cell survival by inhibiting apoptosis and its activation correlates with phosphorylation at Thr308 and Ser473 residues, so the effects of D-limonene on the amount and phosphorylation of Akt were evaluated using western blot analysis. D-limonene did not significantly alter the protein level of Akt, but it decreased the levels of phospho-Akt (Ser473) and phospho-Akt (Thr308) (Fig. 5), which suggested that inactivation of Akt kinase following D-limonene treatment was due to dephosphorylation of Akt, rather than reduction in total Akt protein. We detected the levels of phospho-GSK3 $\beta$ (ser9), which are phosphorylated and inactivated by Akt. Similarly, D-limonene significantly decreased the level of phospho-GSK3 $\beta$ (ser9) (Fig. 5). These results suggested that $\mathrm{D}$-limonene treatment could decrease the activity of Akt.

\section{Discussion}

D-limonene, a monocyclic monoterpene with a lemon-like odor, is reported to have chemopreventive and chemotherapeutic activity in animal models and cultured tumor cells $(14,28,29)$. It has been shown that D-limonene is well tolerated and may have clinical activity in patients with advanced cancer in a phase I clinical trial (9). Moreover, Rabi and Bishayee demonstrated that D-limonene enhanced the antitumor effect of docetaxel against prostate cancer cells without being toxic to normal prostate epithelial cells, which suggested that D-limonene could be used as a potent non-toxic agent to improve the treatment outcome of hormone-refractory prostate cancer with docetaxel (30). However, the molecular mechanism by which D-limonene inhibits the viability of cancer cells has not been established. In this study, we demonstrated that D-limonene inhibited the viability of the LS174T human colon cancer cells in a dose- dependent manner. In particular, we showed that D-limonene induced cell apoptosis, which is partly mediated via the mitochondrial pathway. In addition, we also observed that D-limonene inhibited the PI3K/Akt pathway.

Apoptosis (programmed cell death), is known to be involved in a variety of biological events. Accumulating evidence suggests that most anticancer agents can trigger apoptosis in tumor cells in vivo and in vitro, which appears to be associated with their effectiveness in prevention of tumor growth (31-33). It is well known that caspases play the central role in apoptosis. Caspase- 8 and caspase- 9 are the initiator caspases: caspase- 8 is usually involved in the extrinsic death receptor apoptosis pathway, whereas caspase- 9 has been linked to the intrinsic mitochondrial death pathway. They cleave and activate the downstream effector caspases, such as caspase-3, which cause PARP cleavage and eventually lead to apoptosis. Previous reports indicated that D-limonene may induce cellular apoptosis in some types of cancer $(14,16,30)$. Moreover, in human leukemia cell lines, the caspase-dependent mitochondrial death pathway mediated the D-limonene-induced apoptosis (16). In this study, we showed that caspase activation is also involved in the D-limonene-induced apoptosis. Cleaved caspase-9, caspase-3, and PARP were activated by D-limonene in LS174T cells, but caspase-8 appears not to be involved. Therefore, these results suggest that apoptosis induction by D-limonene might have occurred through a mitochondriamediated pathway.

It is well known that mitochondria play a key role in apoptotic signal transduction in mammalian cells (34). Bcl-2 and Bax are members of the Bcl-2 family that regulates apoptosis by control mitochondria integrity. Although they have highly similar amino acid sequences, their functions are opposed: Bcl-2 acts to inhibit apoptosis, whereas Bax counteracts this effect by heterodimerization with $\mathrm{Bcl}-2$. The ratio of $\mathrm{Bcl} / \mathrm{Bax}$ dictates the sensitivity of cells to a wide variety of apoptotic stimuli (35). In mitochondrion-dependent apoptosis, the disruption of the mitochondrion leads to the release of cytochrome $c$ into the cytosol. The apoptosome containing cytochrome $c$, Apaf-1 and pro-caspase-9 is then assembled, which results in proteolytic processing and activation of procaspase-9. Active caspase-9 in turn activates pro-caspase-3 initiating a caspase signaling cascade to induce apoptosis (36). In the present study, we found that D-limonene decreased the protein level of Bcl-2, but increased the protein level of Bax, which is consistent with previous reports $(16,28)$. We also noted a significant increase of cytochrome $c$ in the cytosol of D-limonene-treated cells. Thus, these results further suggest that D-limonene has the ability to induce the intrinsic mitochondrial apoptosis signaling pathway in LS174T colon cancer cells.

The PI3K/Akt pathway is an important intracellular signaling pathway, which plays a critical role in controlling survival and apoptosis. In many types of cancer this pathway is overactive, supporting cell survival and proliferation (18-20). Several reports have shown that some anticancer agents induce apoptosis, in part, by blocking this pathway (37,38). Activated Akt phosphorylates and inactivates several pro-apoptotic proteins, including BAD (25), and caspase-9 (26), inhibiting the intrinsic apoptotic pathway. Recently, 
geraniol, an acyclic dietary monoterpene, was reported to induce apoptosis by inhibition of AKT signaling (27). Our results showed that D-limonene decreased not only phosphorylated Akt protein levels but also Akt activity. Moreover, we also found that caspase-9, a downstream target of Akt, was cleaved to the active form by the D-limonene treatment. Collectively, these results suggest that inhibition of the Akt pathway contributed, at least in part, to the apoptotic cell death caused by the D-limonene treatment.

In conclusion, the results from the present study suggest that D-limonene inhibited the Akt activation and activated the intrinsic mitochondrial apoptosis signaling pathway in LS174T cells. Although further studies are required to determine whether or not these pathways are involved in the anticancer effect of D-limonene on other cancer cell lines, we believe that this study may provide mechanistic insights into understanding the molecular basis of the anticancer effect of D-limonene.

\section{Acknowledgements}

The present study was supported by the Natural Science Foundation of Heilongjiang Province (D2007-79), the Natural Science Foundation for Young Scientists of Heilongjiang Province (QC2008C23), the Science Research Foundation of the Health Department of Heilongjiang (2006-175) and the Heilongjiang Postdoctoral Science-Research Foundation.

\section{References}

1. Sun J: D-Limonene: safety and clinical applications. Altern Med Rev 12: 259-264, 2007.

2. Hakim IA, Harris RB and Ritenbaugh C: Citrus peel use is associated with reduced risk of squamous cell carcinoma of the skin. Nutr Cancer 37: 161-168, 2000.

3. Maltzman TH, Hurt LM, Elson CE, Tanner MA and Gould MN: The prevention of nitrosomethylurea-induced mammary tumors by d-limonene and orange oil. Carcinogenesis 10: 781-783, 1989.

4. Elson CE, Maltzman TH, Boston JL, Tanner MA and Gould MN Anti-carcinogenic activity of d-limonene during the initiation and promotion/progression stages of DMBA-induced rat mammary carcinogenesis. Carcinogenesis 9: 331-332, 1988.

5. Uedo N, Tatsuta M, Iishi H, et al: Inhibition by D-limonene of gastric carcinogenesis induced by N-methyl-N'-nitro-Nnitrosoguanidine in Wistar rats. Cancer Lett 137: 131-136, 1999.

6. Giri RK, Parija T and Das BR: d-limonene chemoprevention of hepatocarcinogenesis in AKR mice: Inhibition of c-jun and c-myc. Oncol Rep 6: 1123-1127, 1999.

7. Nakaizumi A, Baba M, Uehara $\mathrm{H}$, Iishi $\mathrm{H}$ and Tatsuta $\mathrm{M}$ : d-Limonene inhibits N-nitrosobis(2-oxopropyl)amine induced hamster pancreatic carcinogenesis. Cancer Lett 117: 99-103, 1997.

8. Wattenberg LW, Sparnins VL and Barany G: Inhibition of $\mathrm{N}$-nitrosodiethylamine carcinogenesis in mice by naturally occurring organosulfur compounds and monoterpenes. Cancer Res 49: 2689-2692, 1989.

9. Vigushin DM, Poon GK, Boddy A, et al: Phase I and pharmacokinetic study of D-limonene in patients with advanced cancer. Cancer Research Campaign Phase I/II Clinical Trials Committee. Cancer Chemother Pharmacol 42: 111-117, 1998.

10. Steele VE, Kelloff GJ, Wilkinson BP and Arnold JT: Inhibition of transformation in cultured rat tracheal epithelial cells by potential chemopreventive agents. Cancer Res 50: 2068-2074, 1990.

11. Maltzman TH, Christou M, Gould MN and Jefcoate CR: Effects of monoterpenoids on in vivo DMBA-DNA adduct formation and on phase I hepatic metabolizing enzymes. Carcinogenesis 12 : 2081-2087, 1991.
12. Van der Logt EM, Roelofs HM, van Lieshout EM, Nagengast FM and Peters WH: Effects of dietary anticarcinogens and nonsteroidal anti-inflammatory drugs on rat gastrointestinal UDP-glucuronosyltransferases. Anticancer Res 24: 843-849, 2004.

13. Manuele MG, Barreiro Arcos ML, Davicino R, Ferraro G, Cremaschi G and Anesini C: Limonene exerts antiproliferative effects and increases nitric oxide levels on a lymphoma cell line by dual mechanism of the ERK pathway: relationship with oxidative stress. Cancer Invest 28: 135-145, 2010.

14. Lu XG, Zhan LB, Feng BA, Qu MY, Yu LH and Xie JH: Inhibition of growth and metastasis of human gastric cancer implanted in nude mice by d-limonene. World J Gastroenterol 10: 2140-2144, 2004.

15. Hata T, Sakaguchi I, Mori M, et al: Induction of apoptosis by Citrus paradisi essential oil in human leukemic (HL-60) cells. In Vivo 17: 553-559, 2003.

16. Ji J, Zhang L, Wu YY, Zhu XY, Lv SQ and Sun XZ: Induction of apoptosis by d-limonene is mediated by a caspase-dependent mitochondrial death pathway in human leukemia cells. Leuk Lymphoma 47: 2617-2624, 2006.

17. Franke TF, Hornik CP, Segev L, Shostak GA and Sugimoto C: PI3K/Akt and apoptosis: size matters. Oncogene 22: 8983-8998, 2003.

18. Bader AG, Kang S, Zhao L and Vogt PK: Oncogenic PI3K deregulates transcription and translation. Nat Rev Cancer 5: 921-929, 2005.

19. Niedermeier M, Hennessy BT, Knight ZA, et al: Isoformselective phosphoinositide 3'-kinase inhibitors inhibit CXCR4 signaling and overcome stromal cell-mediated drug resistance in chronic lymphocytic leukemia: a novel therapeutic approach. Blood 113: 5549-5557, 2009.

20. Cui B, Tao J and Yang Y: Studies on the expression patterns of class I PI3K catalytic subunits and its prognostic significance in colorectal cancer. Cell Biochem Biophys 62: 47-54, 2012.

21. Page C, Lin HJ, Jin Y, et al: Overexpression of Akt/AKT can modulate chemotherapy-induced apoptosis. Anticancer Res 20: 407-416, 2000.

22. Opel D, Naumann I, Schneider M, Bertele D, Debatin KM and Fulda S: Targeting aberrant PI3K/Akt activation by PI103 restores sensitivity to TRAIL-induced apoptosis in neuroblastoma. Clin Cancer Res 17: 3233-3247, 2011.

23. Garcia-Echeverria C and Sellers WR: Drug discovery approaches targeting the PI3K/Akt pathway in cancer. Oncogene 27: 5511-5526, 2008 .

24. Crowder RJ and Freeman RS: Glycogen synthase kinase-3 beta activity is critical for neuronal death caused by inhibiting phosphatidylinositol 3-kinase or Akt but not for death caused by nerve growth factor withdrawal. J Biol Chem 275: 34266$34271,2000$.

25. Szanto A, Bognar Z, Szigeti A, Szabo A, Farkas L and Gallyas F Jr: Critical role of bad phosphorylation by Akt in cytostatic resistance of human bladder cancer cells. Anticancer Res 29: 159-164, 2009.

26. Cardone MH, Roy N, Stennicke HR, et al: Regulation of cell death protease caspase-9 by phosphorylation. Science 282: 1318-1321, 1998.

27. Kim SH, Park EJ, Lee CR, et al: Geraniol induces cooperative interaction of apoptosis and autophagy to elicit cell death in PC-3 prostate cancer cells. Int J Oncol 40: 1683-1690, 2012.

28. Chaudhary SC, Siddiqui MS, Athar M and Alam MS: D-Limonene modulates inflammation, oxidative stress and Ras-ERK pathway to inhibit murine skin tumorigenesis. Hum Exp Toxicol 31: 798-811, 2012.

29. Kaji I, Tatsuta M, Iishi H, Baba M, Inoue A and Kasugai H: Inhibition by d-limonene of experimental hepatocarcinogenesis in Sprague-Dawley rats does not involve p21(ras) plasma membrane association. Int J Cancer 93: 441-444, 2001.

30. Rabi T and Bishayee A: d-Limonene sensitizes docetaxelinduced cytotoxicity in human prostate cancer cells: Generation of reactive oxygen species and induction of apoptosis. J Carcinog 8: 9, 2009.

31. Khan N, Adhami VM and Mukhtar H: Apoptosis by dietary agents for prevention and treatment of cancer. Biochem Pharmacol 76: 1333-1339, 2008.

32. Hsieh CC, Hernandez-Ledesma B and de Lumen BO: Lunasin, a novel seed peptide, sensitizes human breast cancer MDA-MB231 cells to aspirin-arrested cell cycle and induced apoptosis. Chem Biol Interact 186: 127-134, 2010. 
33. Sun SY, Hail N Jr and Lotan R: Apoptosis as a novel target for cancer chemoprevention. J Natl Cancer Inst 96: 662-672, 2004.

34. Baysan A, Yel L, Gollapudi S, Su H and Gupta S: Arsenic trioxide induces apoptosis via the mitochondrial pathway by upregulating the expression of Bax and Bim in human B cells. Int J Oncol 30: 313-318, 2007.

35. Uren RT, Dewson G, Chen L, et al: Mitochondrial permeabilization relies on $\mathrm{BH} 3$ ligands engaging multiple prosurvival $\mathrm{Bcl}-2$ relatives, not Bak. J Cell Biol 177: 277-287, 2007.

36. Chen M, Guerrero AD, Huang L, et al: Caspase-9-induced mitochondrial disruption through cleavage of anti-apoptotic BCL-2 family members. J Biol Chem 282: 33888-33895, 2007.
37. Krystal GW, Sulanke G and Litz J: Inhibition of phosphatidylinositol 3-kinase-Akt signaling blocks growth, promotes apoptosis, and enhances sensitivity of small cell lung cancer cells to chemotherapy. Mol Cancer Ther 1: 913-922, 2002.

38. Liu X, Shi Y, Giranda VL and Luo Y: Inhibition of the phosphatidylinositol 3-kinase/Akt pathway sensitizes MDA-MB468 human breast cancer cells to cerulenin-induced apoptosis. Mol Cancer Ther 5: 494-501, 2006. 